\section{Deep impact: deciphering mucosal microbiomes using next-generation sequencing approaches}

Most of the mucosal surfaces that mucosal immunologists study are habitats for a diverse community of commensal microflora that play a profound role in shaping both innate and adaptive immune responses at these mucosal sites. A detailed analysis of these mixed microbial communities is now emerging thanks to the burgeoning field of metagenomics. The unculturable nature of a majority of these organisms and the formidable technical hurdles associated with collecting a sufficiently comprehensive set of sequence data to identify all members of these microbial communities had been the major obstacles impeding progress in metagenomics. The development and subsequent evolution of high-throughput sequencing (HTS) technologies over the past 10 years have spurred explosive growth in metagenomics. Scientists wielding these powerful HTS techniques can harvest a bounty of microbial DNA sequence data at specific mucosal sites for the purposes of either taking a census of the various bacterial species present or striving for the more ambitious goal of identifying many of the genes expressed by the entire resident microflora. ${ }^{1}$

The common feature of the new sequencing technologies that have transformed the landscape for collecting data on mucosal microbiota is reliance on massively parallel "sequencing by synthesis" approaches that are both faster and far less expensive than the classic Sanger dideoxy chain termination method.
At this juncture, the platforms that are being used the most in metagenomics research are based on the use of either a pyrosequencing technique to sequence bead-immobilized DNA fragments in individual drops of a water-in-oil emulsion (the Roche Genome Sequencer FLX platform) or attachment of DNA fragments to a solid phase in a flow cell (the Illumina $1 G$ and Applied Biosystems SOLiD platforms). Choosing which HTS approach is better for a specific metagenomics project requires consideration of multiple factors, including cost per base pair, length of the reads, and accuracy of the individual reads. Each of these HTS approaches generates prodigious amounts of raw sequence data that must be carefully filtered using robust bioinformatics tools to yield informative data. When the main goal of a metagenomics experiment is identification of all the bacterial species present in a sample and their relative abundance, the traditional approach has been sequencing of polymerase chain reaction (PCR) amplicons derived from the variable regions of bacterial $16 \mathrm{~S}$ rDNA.

The first published metagenomic analyses of the human gut microbiota relied entirely on libraries of fecal DNA or PCRamplified 16S rDNA cloned into plasmid vectors followed by conventional DNA sequencing. ${ }^{2,3}$ The pace of progress in characterizing the human gut microbiome accelerated dramatically following the introduction of HTS techniques into the fray. In 2008, Andersson et al. reported an analysis of the microbiota found in human throat, stomach, and fecal samples performed via pyrosequencing of $16 \mathrm{~S}$ rDNA amplicons. ${ }^{4}$ In 2010, investigators from the MetaHIT (Metagenomics of the Human Intestinal Tract) Consortium published an initial "microbial gene catalog" containing most of the prevalent genes in human intestinal microbes; it was generated using Illumina technology to shotgun-sequence large libraries of fragmented DNA recovered from fecal samples of 124 adults. ${ }^{5}$ A 2011 cluster analysis of metagenomic data from fecal samples of 39 individuals in six countries reached the surprising conclusion that all the samples analyzed could be assigned to one of three distinct "enterotypes" identified by the relative predominance of bacteria from three genera (Bacteroides, Prevotella, and Ruminococcus). ${ }^{6}$ Although this observation of a limited number of distinct stable bacterial communities in the human intestine independent of geographic location or diet needs to be further validated in additional cohorts and with nonfecal samples, application of this enterotype concept may simplify future research aimed at identifying correlations between specific gut microbiomes and gastrointestinal health and disease. Shotgun-HTS approaches have also opened the door for identification of novel pathogens besides bacteria in mucosal samples; for example, pyrosequencing of human stool samples has yielded several novel human picornaviruses. ${ }^{7,8}$ The value of metagenomic analysis using HTS methods also extends to mouse models. Some knockout mice lacking host proteins involved in the sensing of bacteria (such as TLR5 and NLRP6) exhibit significant shifts in the composition of their resident enteric microbiota that are associated with the development of colitis. ${ }^{9,10}$

The application of HTS methods to address key experimental questions in mucosal immunology is still in its infancy. Further refinements in these technologies and continued declines in their cost are likely to make them increasingly useful experimental tools.

\section{Ifor R Williams}

(C) 2011 Society for Mucosal Immunology

\section{REFERENCES}

1. Petrosino, J.F., Highlander, S., Luna, R.A., Gibbs, R.A. \& Versalovic, J. Metagenomic pyrosequencing and microbial identification. Clin. Chem. 55, 856-866 (2009).

2. Gill, S.R. et al. Metagenomic analysis of the human distal gut microbiome. Science $\mathbf{3 1 2}$ 1355-1359 (2006). 
3. Kurokawa, K. et al. Comparative metagenomics revealed commonly enriched gene sets in human gut microbiomes. DNA Res. 14, 169-181 (2007).

4. Andersson, A.F. et al. Comparative analysis of human gut microbiota by barcoded pyrosequencing. PLoS One 3, e2836 (2008).

5. Qin, J. et al. A human gut microbial gene catalogue established by metagenomic sequencing. Nature 464, 59-65 (2010).

6. Arumugam, M. et al. Enterotypes of the human gut microbiome. Nature 473, 174-180 (2011).

7. Kapoor, A. et al. A highly prevalent and genetically diversified Picornaviridae genus in South Asian children. Proc. Natt. Acad. Sci. USA 105, 2048220487 (2008).

8. Greninger, A.L. et al. The complete genome of klassevirus - a novel picornavirus in pediatric stool. Virol. J. 6, 82 (2009).

9. Vijay-Kumar, M. et al. Metabolic syndrome and altered gut microbiota in mice lacking Toll-like receptor 5. Science 328, 228-231 (2010).

10. Elinav, E. et al. NLRP6 inflammasome regulates colonic microbial ecology and risk for colitis. Cell 145, 745-757 (2011). 